EPJ Web of Conferences 41, 05046 (2013)

DOI: $10.1051 /$ epjconf/20134105046

(C) Owned by the authors, published by EDP Sciences, 2013

\title{
Ultrafast inter-ionic charge transfer of transition-metal complexes mapped by femtosecond $x$-ray powder diffraction
}

\author{
F. Zamponi ${ }^{1, a}$, B. Freyer ${ }^{1}$, V. Juvé ${ }^{1}$, J. Stingl ${ }^{1}$, M. Woerner ${ }^{1}$, M. Chergui ${ }^{2}$, and T. Elsaesser ${ }^{1}$ \\ 1 Max-Born-Institut für Nichtlineare Optik und Kurzzeitspektroskopie, D-12489 Berlin, Germany \\ 2 Laboratory of Ultrafast Spectroscopy, EPF Lausanne, CH-1015 Lausanne, Switzerland
}

\begin{abstract}
Transient electron density maps are derived from $\mathrm{x}$-ray diffraction patterns of photoexcited $\left[\mathrm{Fe}(\mathrm{bpy})_{3}\right]^{2+}\left(\mathrm{PF}_{6}^{-}\right)_{2}$ powder. Upon photoexcitation, the ${ }^{5} \mathrm{~T}_{2}$ quintet state reveals a charge transfer from the $\mathrm{PF}_{6}^{-}$ions and from the Fe atoms to neighboring bpy units. The charge transfer from the Fe points to a partial and weak charge-transfer character of this state.
\end{abstract}

Transition-metal complexes have raised strong interest as model systems for intramolecular electron transfer from the metal to the ligand and for applications as photosensitizers in a variety of energy conversion devices as well as building blocks for the next generation of computer memories (optical writing / magnetic reading) [1,2]. Ultrafast studies of basic photophysical properties and processes of such complexes have mainly focused on dilute liquid solutions where the complexes and their counterions are spatially separated by their individual solvation shells. Numerous applications require, however, a high packing density of the complexes in the solid state where interactions between complexes and their neighboring counterions as well as between different complexes may modify the transient behavior substantially.

In this paper, we present the first femtosecond $\mathrm{x}$-ray diffraction study of crystalline $\mathrm{Fe}(\mathrm{bpy})_{3}\left(\mathrm{PF}_{6}\right)_{2}$ (iron(II)-tris-bipyridine hexafluorophosphate, Fig. 1a), a transition-metal complex consisting of the $\left[\mathrm{Fe}(\mathrm{bpy})_{3}\right]^{2+}$ cations and two $\mathrm{PF}_{6}^{-}$anions. Photoexcitation from the low-spin ground state into the singlet Metal-to-Ligand Charge Transfer (MLCT) states initiates an ultrafast cascade of intersystem crossing and vibronic relaxation processes that leads to the formation of the lowest excited ${ }^{5} \mathrm{~T}_{2}$ quintet state $[1,2]$. The quintet state decays non-radiatively with a lifetime of approximately $650 \mathrm{ps}$ back to the low-spin ground state. X-ray powder diffraction patterns of the electronically excited complexes recorded with a $100 \mathrm{fs}$ time resolution allow to retrieve transient maps of electron density. Such maps reveal a hitherto unknown charge transfer from the $\mathrm{PF}_{6}^{-}$anions and from the Fe centers to the bipyridine moieties of the complexes.

The centrosymmetric crystal structure of $\mathrm{Fe}(\mathrm{bpy})_{3}\left(\mathrm{PF}_{6}\right)_{2}$ is trigonal and belongs to the space group $\mathrm{P} \overline{3} \mathrm{c} 1$ with two formula units per unit cell (see Fig. 1a for a top view). In the experiments, a crystalline powder of $\left[\mathrm{Fe}(\mathrm{bpy})_{3}\right]^{2+}\left(\mathrm{PF}_{6}^{-}\right)_{2}$ which is held between $20 \mu \mathrm{m}$ thick diamond windows is excited by a sub-40 fs pulse at $800 \mathrm{~nm}$. The pump pulse populates a MLCT state but also resonant dd states (metal centered) via a 2-photon absorption. Transient powder diffraction patterns are generated with a $100 \mathrm{fs}$ hard $\mathrm{x}$-ray probe pulse $(\mathrm{Cu}-\mathrm{K} \alpha$, wavelength $0.154 \mathrm{~nm})$ and recorded with a large area X-ray detector at different pump-probe delays [3-5]. Transient electron density maps were derived from the diffraction patterns with the help of the maximum entropy method [6].

An x-ray diffraction pattern consisting of approximately 20 Debye Scherrer rings is shown in the inset of Fig. 1b. A lineout of such patterns recorded with a total acquisition time of 8 hours is shown in Fig. $1 \mathrm{~b}$ where the $\mathrm{x}$-ray intensity integrated over individual diffraction rings is plotted as a function of the diffraction angle. Upon optical excitation, the ring pattern displays intensity changes of up to

a e-mail: zamponi@mbi-berlin.de

This is an Open Access article distributed under the terms of the Creative Commons Attribution License 2.0, which permits unrestricted use, distribution, and reproduction in any medium, provided the original work is properly cited. 

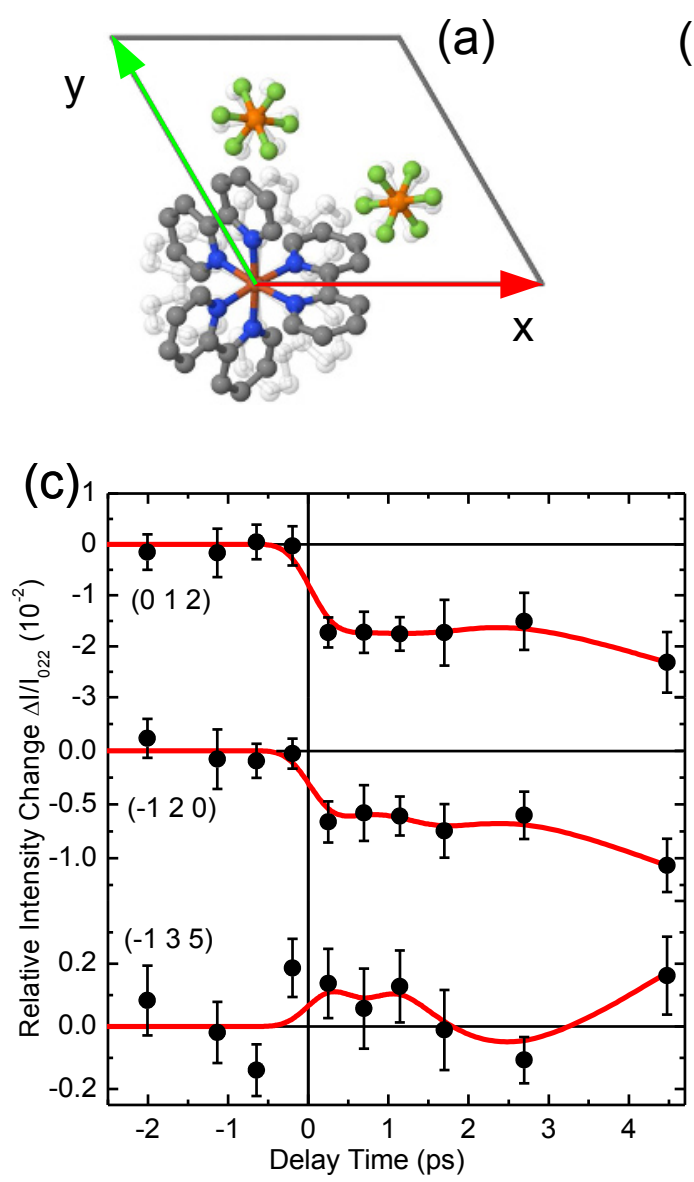
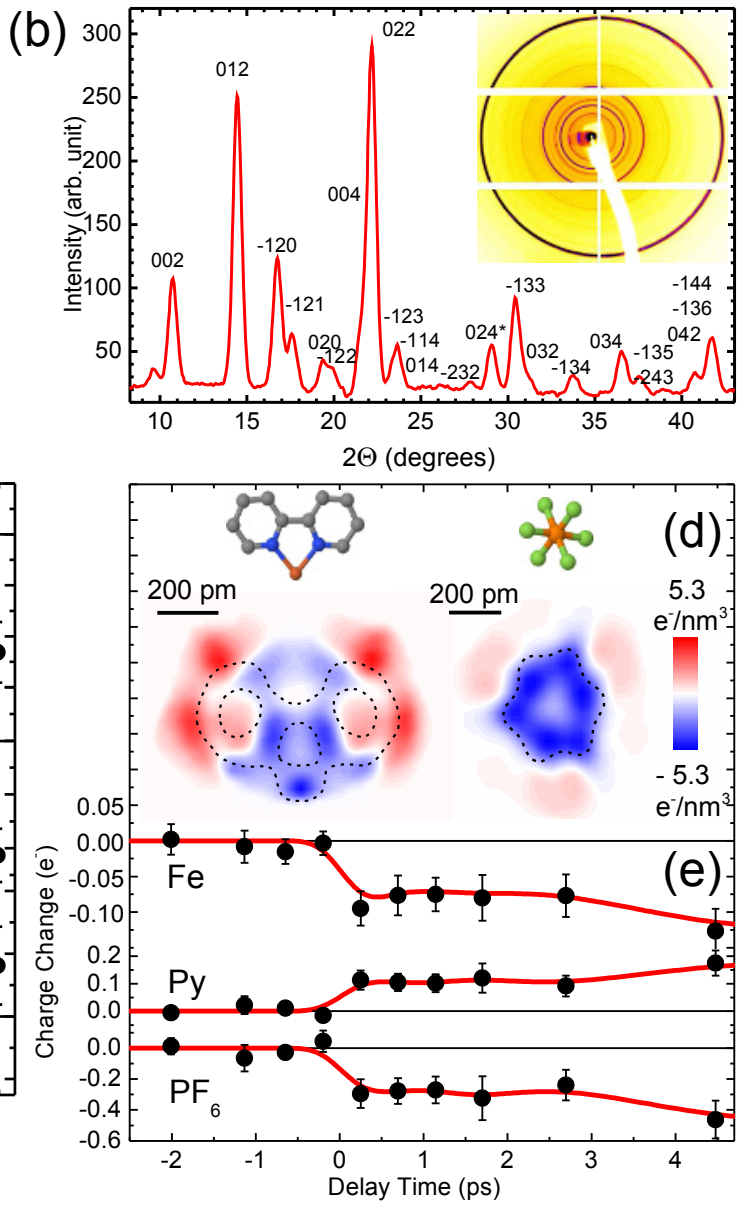

Fig. 1. Fig 1. (a) Unit cell of $\mathrm{Fe}(\mathrm{bpy})_{3}\left(\mathrm{PF}_{6}\right)_{2}$. Fe atoms are brown, $\mathrm{N}$ blue, $\mathrm{C}$ gray, $\mathrm{F}$ green, and $\mathrm{P}$ orange. (b) Powder diffraction pattern as recorded with the x-ray plasma source, with an integration time of 8 hours. The signal integrated along the rings (cf. inset) is plotted as a function of the diffraction angle $2 \theta$. The Miller indices of the strongest reflections are shown. Inset: Diffraction pattern as recorded with the x-ray detector. (c) Change of the diffracted intensity, normalized to the steady state intensity of the 022 diffraction ring, as a function of delay time between optical pump and x-ray probe for three selected reflections. (d) Electron density maps of the quintet state derived from the diffraction data for a delay time of $250 \mathrm{fs}$. The contour plots were obtained in the following way: the whole unit cell volume was divided in sub-volumes, each containing one atom (H atoms were not taken into account). Sub-volumes were grouped together to build the bipyridines and the anions. Finally, the electron density changes of the sub-volumes were projected on a plane parallel to the plane containing the Fe atom and the bipyridine (left) and on a plane perpendicular to the z-axis for the anion (right). A ball-and-stick model of cation and anion is also shown. The black dashed line is a contour line of the stationary electron density, indicating the position of the the iron atom and one bi-pyridine molecule and of the six fluorine atoms and the phosphor atom. (e) Time evolution of the total charge change on the $\mathrm{Fe}$, bpy, and anion as a function of pump-probe delay.

$2 \%$. In Fig. 1c, the change of diffracted intensity on 3 selected reflections is plotted as a function of pump-probe delay. The transients display intensity changes rising within the $100 \mathrm{fs}$ time resolution of the experiment and persisting into the picosecond time domain. The fast rise is consistent with the sub-150 fs population time of the quintet state observed in solution-phase measurements [1].

Electron density maps derived from diffraction patterns recorded at a delay time of 250 fs concerning the ${ }^{5} \mathrm{~T}_{2}$ quintet state are presented in Fig. 1d. The change of electron density $\Delta \rho$ is shown in the plane containing the $\mathrm{Fe}$ atom and a bipyridine unit and for a projection of the $\mathrm{PF}_{6}^{-}$ions on the xy-plane. Fig. 1d gives evidence of a charge transfer from the Fe atom to the bypyridine unit. On the 
latter, the charge density is further enhanced by a transfer from the $\mathrm{PF}_{6}^{-}$counterions. The time evolution and the absolute value of $\Delta \rho$ on the different moieties is plotted in Fig. 1e. It should be noted that the amount of charge transferred from the counterions to the bipyridine is substantially higher than the amount originating from the Fe atom, as one would expect since in the quintet state the Fe is back to the $2+$ oxidation state.

The persistence of charge transfer from the Fe atoms many picoseconds after the optical excitation reveals a partial and weak charge-transfer character of the quintet state which may explain the ultrafast $\mathrm{MLCT}-{ }^{5} \mathrm{~T}_{2}$ relaxation [1,2]. Our results demonstrate that Coulomb-mediated nearest neighbor interactions in the crystalline phase have a strong influence on the charge density distribution of transition-metal complexes.

\section{References}

1. A. Cannizzo, C.J. Milne, C. Consani, W. Gawelda, C. Bressler, F. Van Mourik, and M. Chergui, Coord. Chem. Rev. 254, (2010) 2677.

2. C. Bressler, C. Milne, V. T. Pham, A. ElNahhas, R. M. Van der Veen, W. Gawelda, S. L. Johnson, P. Beaud, D. Grolimund, M. Kaiser, and others, Science 323, (2009) 5913.

3. M. Woerner, F. Zamponi, Z. Ansari, J. Dreyer, B. Freyer, M. Premont-Schwarz and T. Elsaesser, J. Chem. Phys. 133, (2010) 064509.

4. F. Zamponi, Z. Ansari, M. Woerner and T. Elsaesser, Optics Express 18, (2010) 948.

5. F. Zamponi, P. Rothhardt, J. Stingl, M. Woerner and T. Elsaesser, PNAS 109, (2012) 5207.

6. S. van Smaalen, L. Palatinus and M. Schneider, Acta Cryst. A 59, (2003) 459. 